\title{
Cramming is not learning
}

\author{
Shakirat O. Oyetunji, MD, and Nahush A. Mokadam, MD
}

From Cardiothoracic Surgery, University of Washington Medical Center, Seattle, Wash.

Disclosures: Dr Mokadam is a consultant and investigator for Medtronic, Abbott, and SynCardia. Dr Oyetunji has nothing to disclose with regard to commercial support.

Received for publication March 30, 2018; accepted for publication April 5, 2018; available ahead of print May 9, 2018.

Address for reprints: Nahush A. Mokadam, MD, 1959 NE Pacific St, AA-115 Box 356310, Seattle, WA 98195 (E-mail: mokadamn@uw.edu).

J Thorac Cardiovasc Surg 2018;156:928

$0022-5223 / \$ 36.00$

Copyright (C) 2018 by The American Association for Thoracic Surgery

https://doi.org/10.1016/j.jtcvs.2018.04.009

\begin{abstract}
If the license to practise meant the completion of his education how sad it would be for the practitioner, how distressing to his patients! More clearly than others the physician should illustrate the truth of Plato's saying - that education is a life-long process. ${ }^{.}$
\end{abstract} — Sir William Osler, 1900

Trainees in thoracic surgery are challenged by the plethora of educational resources available across different platforms along with ever-present time constraints. There has been an emphasis over the past decade on how best to train adult learners to most efficiently acquire the required knowledge and to develop lifelong learning strategies. The national web-based Thoracic Surgery Curriculum (TSC) has been 1 such effort aimed specifically at thoracic surgery trainees. ${ }^{2}$ In contrast to Plato's philosophical openair school in the grove of Academus, the TSC is electronic and standardized. Although it has its critics, it is comprehensive and currently the gold standard in North America.

Luc and colleagues ${ }^{3}$ set out to characterize study habits of thoracic surgery trainees and examine the relationship between TSC login frequency and improvement on the thoracic surgery in-training examination (TSITE). Cramming (ie, more frequent logins) did not improve TSITE scores in the overall cohort; however, individual trainees with the highest use during the month leading up to the TSITE improved relative to their own cohort. Similarly, the report by Giordano and colleagues ${ }^{4}$ on a predictive model for performance on the US Medical Licensing Examination (USMLE) Step 1 showed no statistically significant relationship between the number of weeks spent studying and USMLE Step 1 score. They did find a trend of increasing scores in a cohort of students who had not obtained straight A's during the first 2 years of medical school if they had studied longer than 6 weeks. ${ }^{4}$ The study by Giordano and colleagues ${ }^{4}$ supports the findings of Luc and colleagues ${ }^{3}$ : Intensification of study habits has some value to some learners in test preparation, especially those for whom a wider knowledge gap exists.

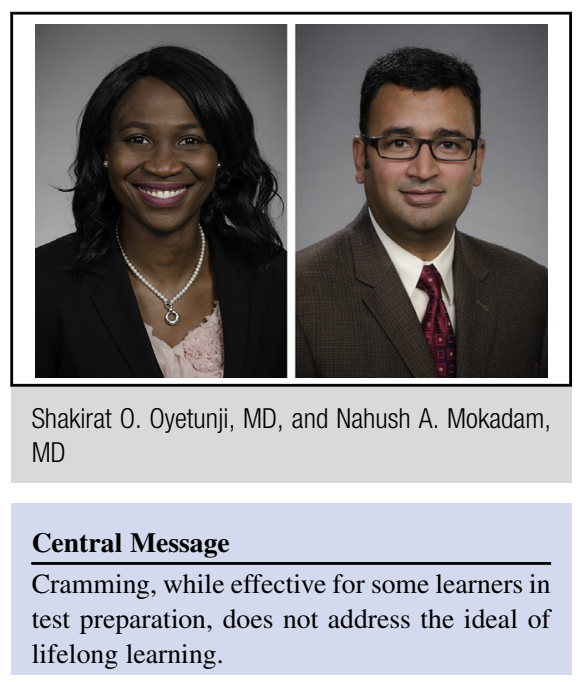

See Article page 922.

Editors of the TSC, like one of the authors (N.A.M.), consider any measure of increased curriculum use to be a success! Surely the report by Luc and colleagues ${ }^{3}$ reflects well on the nationwide and sustained effort needed to create the TSC, and validates the use of the curriculum in TSITE preparation. However, as educators we should limit our enthusiasm. Other elements of the TSC, such as quiz performance (and frequency), offline studying, and group studying could not be measured by the existing software platform. Nor is there a measure of lifelong learning principles, such as repetition, critical thinking, case review, or formative feedback. This is the next frontier in our assessment of the TSC, and may be more deliverable with the transition in software platform. Ideally, we would be able to demonstrate that the TSC is not only a tool for preparation for exams like the TSITE but also, more importantly, a vehicle for lifelong learning. Like Osler, ${ }^{1}$ we hope to propagate Plato's philosophy.

\section{References}

1. Osler W. An address on the importance of post-graduate study: delivered at the opening of the Museums of the Medical Graduates College and Polyclinic. BMJ. 1900;2:73-5.

2. The thoracic surgical curriculum. Available at: https://learnctsurgery.sts.org/lms/ pages/about_us. Accessed March 22, 2018.

3. Luc J, Verrier E, Allen M, Aloia L, Baker C, Fann JI, et al. Does cramming work? Impact of national web-based thoracic surgery curriculum login frequency on thoracic surgery in-training exam performance. J Thorac Cardiovasc Surg. 2018;156:922-7.

4. Giordano C, Hutchinson D, Peppler R. A predictive model for USMLE Step 1 scores. Cureus. 2016;8:e769. 